Article

\title{
Functional Expression and Characterization of the Recombinant $N$-Acetyl-Glucosamine/ $N$-Acetyl- Galactosamine-Specific Marine Algal Lectin BPL3
}

\author{
Hyun-Ju Hwang ${ }^{1}$, Jin-Woo Han ${ }^{1}$, Gwang Hoon Kim ${ }^{2, *}$ and Jong Won Han ${ }^{1, *}$ \\ 1 Department of Genetic Resources Research, National Marine Biodiversity Institute of Korea, \\ Seocheon 33662, Korea; hjhwang@mabik.re.kr (H.-J.H.); hiclow@mabik.re.kr (J.-W.H.) \\ 2 Department of Biology, Kongju National University, Kongju 32588, Korea \\ * Correspondence: ghkim@kongju.ac.kr (G.H.K.); jwhan@mabik.re.kr (J.W.H.); \\ Tel: +82-41-850-8504 (G.H.K.); +82-41-950-0760 (J.W.H.); Fax: +82-41-950-0765 (J.W.H.)
}

Received: 28 November 2017; Accepted: 28 December 2017; Published: 5 January 2018

\begin{abstract}
Lectins, characterized by their carbohydrate-binding ability, have extensive practical applications. However, their industrial use is limited due to impurity. Thus, quality-controlled production of recombinant lectin is necessary. In this study, the algal lectin BPL3 (Bryopsis plumosa lectin 3) was successfully produced using a bacterial expression system, BL21(DE3), with an artificial repeated structure (dimeric construct). Recombinant dimeric BPL3 (rD2BPL3) was confirmed by LC-MS/MS spectrometry. Expression efficiency was greater for the construct with the repeat structure (rD2BPL3) than the monomeric form (rD1BPL3). Optimal conditions for expression were $1 \mathrm{mM}$ IPTG at $20{ }^{\circ} \mathrm{C}$. Recombinant lectin was purified under denaturing conditions and refolded by the flash dilution method. Recombinant BPL3 was solubilized in $1 \times$ PBS containing $2 \mathrm{M}$ urea. rD2BPL3 showed strong hemagglutination activity using human erythrocyte. rD2BPL3 had a similar sugar specificity to that of the native protein, i.e., to $\mathrm{N}$-acetyl-glucosamine (GlcNAc) and $\mathrm{N}$-acetyl-galactosamine (GalNAc). Glycan array results showed that recombinant BPL3 and native BPL3 exhibited different binding properties. Both showed weak binding activity to $\alpha$-Man-Sp. Native BPL3 showed strong binding specificity to the alpha conformation of amino sugars, and rD2BPL3 had binding activity to the beta conformation. The process developed in this study was suitable for the quality-controlled large-scale production of recombinant lectins.
\end{abstract}

Keywords: Bryopsis plumosa; BPL3; lectin; hemagglutinin; recombinant; tandem repeat; GlcNAc; GalNAc

\section{Introduction}

Lectins are well-known carbohydrate-binding proteins able to agglutinate cells by glycoconjugation; they have many medical and scientific applications [1]. For example, lectins are a potential diagnostic molecule for carbohydrate profiling on cell surfaces [2] and can be used for the identification of glycoproteins [3]. Fluorescently labelled lectins have been used for the visualization of polysaccharides in biofilms of Pseudomonas aeruginosa [4]. Lectin affinity chromatography has become a common method for the isolation of glycoproteins from cell extracts [5]. Recently, the application of silver nanoparticles with C-type lectin as a recognition ligand has been suggested for bacterial detection [6]. Lectin histochemistry has also be used for the diagnosis of Sida carpinifolia (Malvaceae) poisoning in sheep [7]. An efficient strategy for the systematic production of recombinant lectins for use in microarray technology has also been described [8].

The utility of GalNAc-specific lectins has been reported by several research groups. Gal/GalNAc-specific lectin is a vaccine candidate for amoebiasis and a focus of immunogenicity 
studies [9]. Wisteria floribunda agglutinin (WFA), a GalNAc-specific lectin, shows promise for cancer biomarker detection, with disaccharide LacdiNAc $(\beta-D-G a l N A c-[1 \rightarrow 4]-D-G l c N A c)$ recognition properties [10].

To date, approximately 800 algal species have been screened and approximately $60 \%$ of these taxa show lectin activity [11]. However, only a few algal lectins (about 50 lectins from marine algae) have been isolated and characterized owing to interfering substances, such as polyphenols, in algae. Insufficient algal biomass is another barrier to the application and commercialization of algal lectin [11]. To overcome these limitations, recombinant techniques are a potentially useful tool for the production and biochemical characterization of active algal lectins.

BPL3 is a previously isolated GlcNAc/GalNAc-specific lectin [12]. This protein and other B. plumosa-derived lectins (Bryohealin and BPL4) have important functions in the wound healing process of B. plumosa during protoplast regeneration from mechanically damaged cells [12,13]. BPL3 is similar to H-type lectin, which is produced by invertebrates, and not by plants [12]. Based on comparative sequence analyses and the conservation of active sites between BPL3 and the $\mathrm{H}$ lectin group, BPL3 was suggested as a research tool in various fields within biochemical and medical sciences [12]. These sequence analyses also suggest that BPL3 is an example of parallel evolution across species boundaries. Despite its overall importance, its biochemical properties, including active sites, are still unclear owing to inability to produce high quantities of pure protein. The production of recombinant BPL3 has not been reported.

Most plants and algae have a heterogeneous mixture of lectin isoforms with diverse biological activities; therefore, a lectin isolated from natural sources is typically not preferred for medical applications [14,15]. In addition, the inability to obtain large amounts of lectins from natural sources is a major hurdle for medical uses. The production of lectins by recombinant techniques was a major break-through, but production of the active form is difficult using bacterial expression systems [16]. Many plant lectins have a dimeric or multimeric structure with homologous subunits exhibiting covalent or non-covalent interactions, and this is demanding in bacterial expression systems. It requires the precise optimization of hydrogen or salt concentrations, which may be un-controllable and difficult to reproduce.

Tandem repeat domain structures have been reported in native lectin from Silurus asotus eggs [17], mannose-binding lectin from Boodlea coacta [18], and lectin from Aglaothamnion callophyllidicola [19]. For example, rhodobindin, a lectin produced from the red alga A. callophyllidicola involved in the cell-cell recognition process during sexual reproduction [19], consists of an internal tandem repeat structure with at least eight domains. The tandem repeat structure contributes to the production of the active protein and influences recombinant expression [14].

Based on these previous results for rhodobindin, we predicted that the construction of internal tandem repeat domains may be useful for the production of active lectin. In this study, active recombinant BPL3 was produced with artificial internal tandem repeat domains and its biochemical properties were characterized. The potential applications of this recombinant lectin for biochemical and medical research are discussed.

\section{Results}

\subsection{Cloning of rBPL3}

BPL3 cDNA was codon-optimized to avoid codon mismatches between marine green alga and bacterial tRNA (Figure 1). The expression efficiency of un-optimized cDNA was inadequate in normal conditions $\left(37^{\circ} \mathrm{C}\right.$, overnight incubation; data not shown), but codon-optimized BPL3 cDNA was expressed (Figure 2). To determine the effect of a repeated sequence array of homologous domains on hemagglutination activity and expression efficiency, monomeric (rD1BPL3) and dimeric (rD2BPL3) sequences were constructed. The expression efficiency of $\mathrm{rD2BPL3}$, i.e., the dimeric form, was more than 10-fold greater than that of the monomeric form of rBPL3, rD1BPL3 (Figure 2). 
(A)

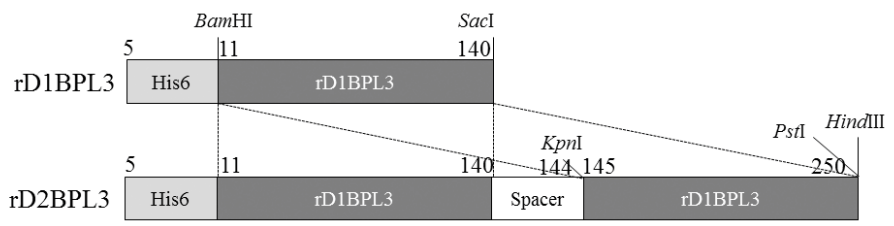

(B)

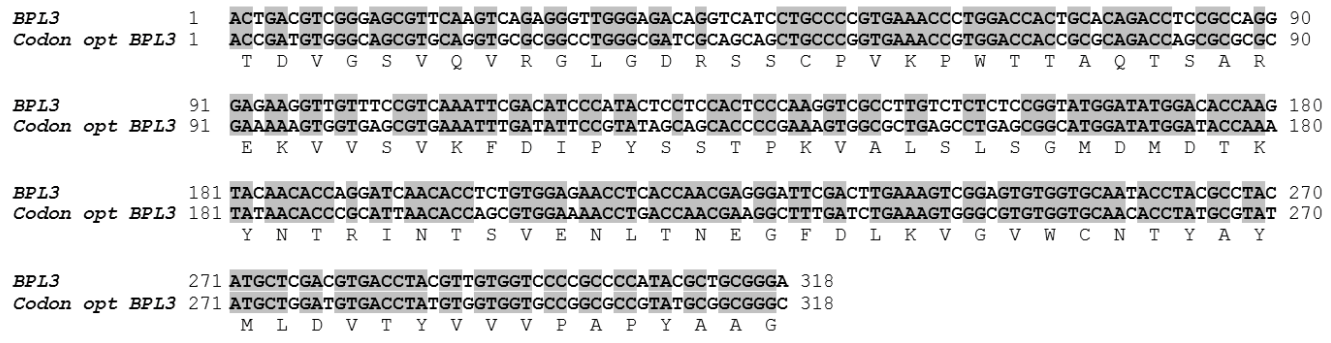

Figure 1. Construction of rD1BPL3 and rD2BPL3. (A) Monomeric and dimeric forms of rBPL3; (B) Codon-optimized BPL3.

\section{(A)}

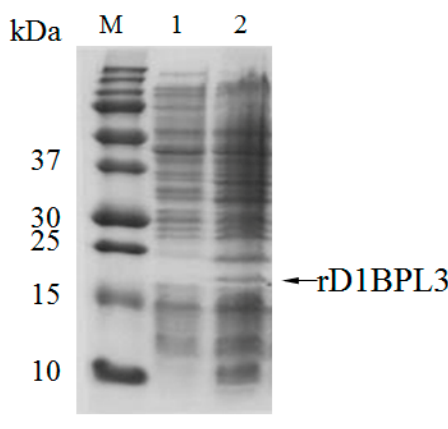

(B)

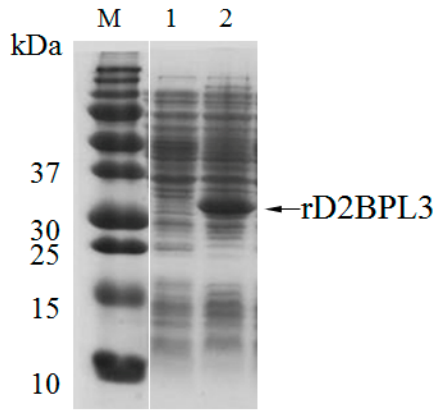

(C)

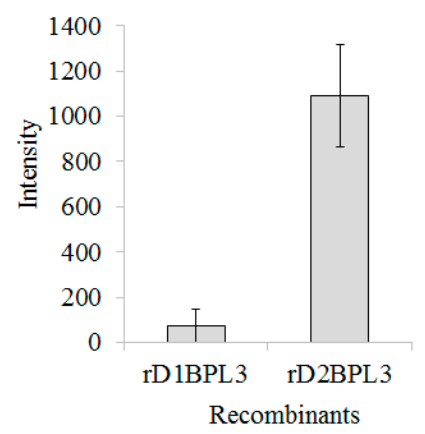

Figure 2. Expression efficiency of rD1BPL3 and rD2BPL3 according to repeated sequences. (A) rD1BPL3 expressed in BL21(DE3), (B) rD2BPL3 expressed in BL21(DE3). M, Molecular weight marker; Lane 1, un-induced lysate; Lane 2, IPTG-induced lysates. Arrows indicate target proteins. (C) Comparison of target protein intensity depending on the repeat sequence array. Error bars indicate standard deviation. $n=5$.

\subsection{Selection of an Expression Host and Optimization of Conditions}

BL21(DE3) was chosen as an expression host for rD2BPL3; other bacterial hosts showed similar expression patterns to that of BL21(DE3) at $37{ }^{\circ} \mathrm{C}$ (Supplementary Figure S1).

The expression efficiency of recombinant lectin was not highly affected by IPTG (isopropyl- $\beta$-Dthiogalactopyranoside) at various concentrations at $37^{\circ} \mathrm{C}$. The protein expression level with $1 \mathrm{mM}$ IPTG was about 1.5-fold higher than those for the other conditions, but all expression levels were within the margin of error (Figure 3A). Temperature was an essential factor for protein induction. The expression of rD2BPL3 was highest at $25^{\circ} \mathrm{C}$ and lowest at $30^{\circ} \mathrm{C}$ after $7 \mathrm{~h}$ of induction. Increased induction durations influenced the production of rD2BPL3 at $20^{\circ} \mathrm{C}$ and $25{ }^{\circ} \mathrm{C}$, but not at normal temperatures (i.e., $30^{\circ} \mathrm{C}$ and $37^{\circ} \mathrm{C}$ ) (Figure 3B). All expression hosts produced inclusion bodies in the insoluble form in the tested conditions (Supplementary Figure S1). 
(A)

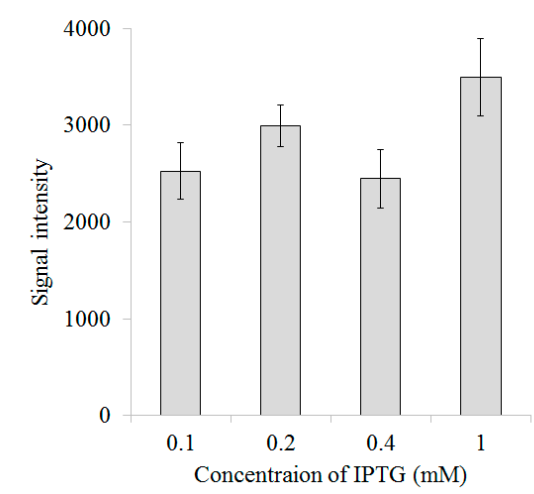

(B)

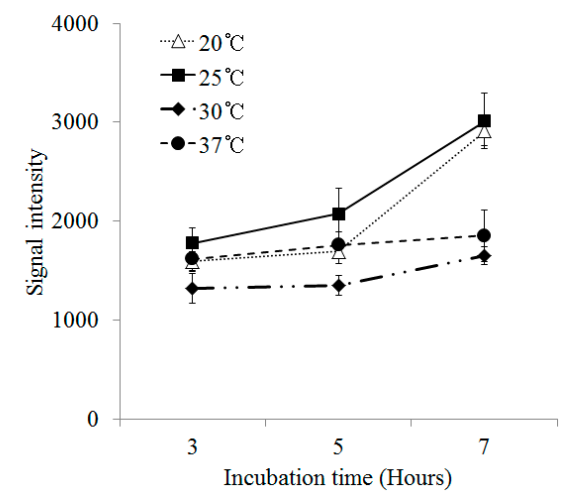

Figure 3. Expression efficiency of recombinant lectin in various conditions. (A) Induction efficiency for various concentrations of IPTG (at $37^{\circ} \mathrm{C}, \mathrm{OD}_{600}=0.4-0.6$, overnight); (B) The effects of temperature $\left(20-37^{\circ} \mathrm{C}\right)$ and incubation time $(3-7 \mathrm{~h})$ on the induction of recombinant protein $\left(\mathrm{OD}_{600}=0.4-0.6\right.$, $0.4 \mathrm{mM}$ IPTG).

\subsection{Purification of Recombinant Lectins}

Both rD1BPL3 and rD2BPL3 were solubilized in denaturing conditions (8 M urea in PBS), but not in PBS alone (lacking urea). Ni-NTA agarose was used for the purification of recombinant lectins. Most recombinant lectins were bound to the affinity matrix. Recombinant lectin was eluted by a stepwise gradient of $75 \mathrm{mM}, 125 \mathrm{mM}$, and $250 \mathrm{mM}$ imidazole (Figure 4A). A single band was observed from the eluted fraction for each recombinant (Figure 4B,C). Approximately $3 \mathrm{mg}$ of rD2BPL3 was obtained from $1 \mathrm{~L}$ bacterial culture, while rD1BPL3 was obtained in sufficient quantities for further analysis despite the presence of a purified band in Figure 4C. The amino acid sequence of the recombinant protein was confirmed by LC-MS/MS spectrometry (Supplementary Figure S2).

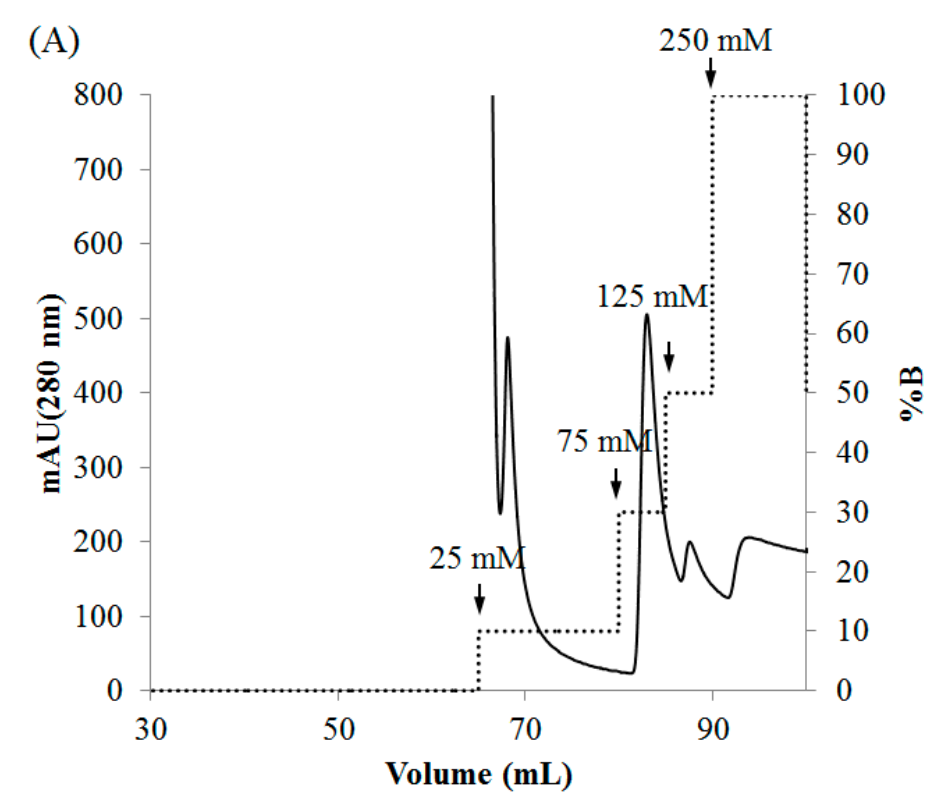

(B)

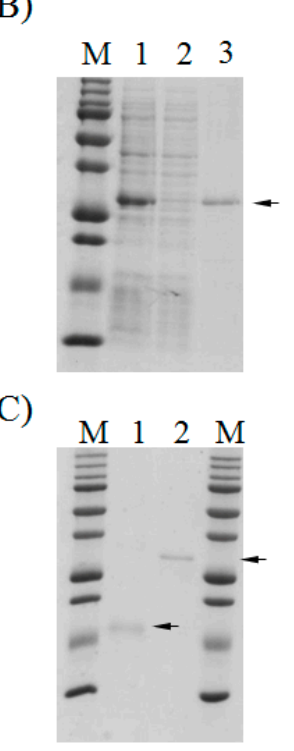

Figure 4. Purification of recombinant lectins by Ni-NTA agarose chromatography. (A) Chromatogram showing protein elution from the column; (B,C) SDS-PAGE; (B) Purification of rD2BPL3, M, molecular weight marker; lane 1, crude extract; lane 2, flow-through fraction; Lane 3, purified rD2BPL3; (C) Purified recombinant lectins; M, Molecular weight marker; lane 1, rD1BPL3; lane 2, rD2BPL3. 
The flash dilution method was effective for rD2BPL3 refolding. The dialysis method accelerated the production of inclusion bodies; the protein formed inclusion bodies, when the dialysis buffer contained less than $4 \mathrm{M}$ urea (data not shown). Purified proteins were diluted in 0.2 to $4 \mathrm{M}$ urea in PBS buffer. The hemagglutination activity when the protein was diluted in $2 \mathrm{M}$ urea was 16-fold greater than that in the $8 \mathrm{M}$ urea condition (Figure 5A). The protein was precipitated and a loss of activity was observed at less than $2 \mathrm{M}$ urea (Figure 5). The optimal concentration of urea for refolding was $2 \mathrm{M}$ (Figure 5).

(A)

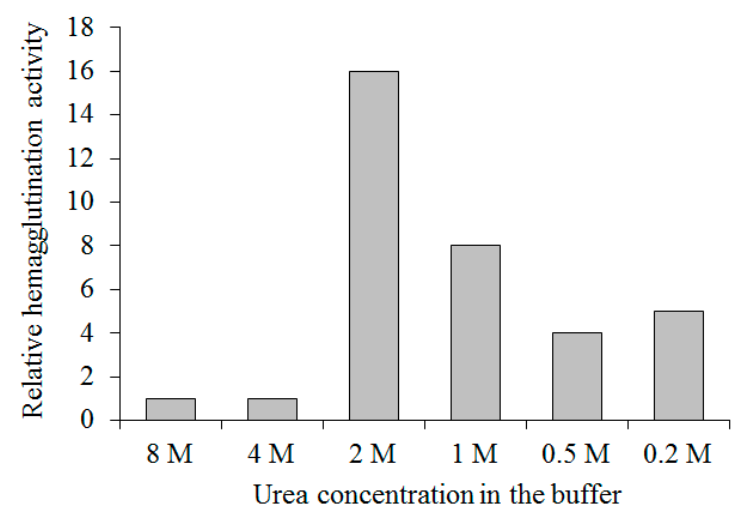

(B)

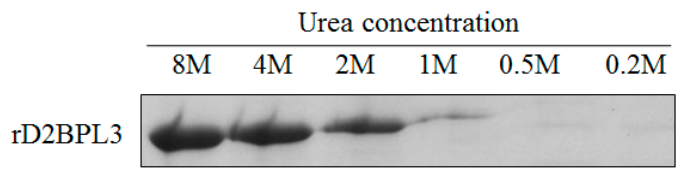

Figure 5. Refolding of rD2BPL3 inclusion bodies (IBs) by the flash refolding method. (A) Relative hemagglutination activity of rD2BPL3 at various concentration of a denaturant, urea $(0.2-8 \mathrm{M})$; (B) SDS-PAGE, solubility of rD2BPL3 using various concentrations of urea. After flash refolding, insoluble components were removed by centrifugation and the supernatant was subjected to SDS-PAGE. See Materials and Methods section.

\subsection{Carbohydrate Specificity and Heat-Stability of Recombinant Lectin}

The recombinant lectin rD2BPL3 showed agglutination activity using human blood cells. The minimum concentration of purified rD2BPL3 required for agglutination was $12.5 \mu \mathrm{g} / \mathrm{mL}$ (Figure 6A), while that of native BPL3 was $0.78 \mu \mathrm{g} / \mathrm{mL}$. rD1BPL3 did not show agglutination activity. The hemagglutination activity was clearly inhibited by pre-incubation with $N$-acetyl-D-glucosamine, $N$-acetyl-D-galactosamine, and fetuin, similar to the results obtained for native BPL3 (Figure 6B). The minimum inhibitory concentration of GlcNAc was $62.5 \mathrm{mM}$ and that of GalNAc was $31.3 \mathrm{mM}$, corresponding to those of the native form of BPL3 (Table 1). rD2BPL3 also showed similar heat-stability to that of native BPL3 and the protein did not require divalent ions to maintain its sugar binding activity (data not shown). 
Table 1. Inhibition of hemagglutination activity of native BPL3 and rD2BPL3 by various substances.

\begin{tabular}{ccc}
\hline \multirow{2}{*}{ Substance } & \multicolumn{2}{c}{ Minimum Inhibitory Concentration } \\
\cline { 2 - 3 } & Native BPL3 & rD2BPL3 \\
\hline Fetuin & $19.53 \S$ & $4.88 \S$ \\
D-Mannose & $\mathrm{NI}$ & $\mathrm{NI}$ \\
L-Fucose & $\mathrm{NI}$ & $\mathrm{NI}$ \\
D-Fructose & $\mathrm{NI}$ & $\mathrm{NI}$ \\
$\beta$-Lactose & $\mathrm{NI}$ & $\mathrm{NI}$ \\
$N$-acetyl-D-glucosamine & 125 & 62.5 \\
$N$-acetyl-D-galactosamine & 62.5 & 31.25 \\
D-Galactose & $\mathrm{NI}$ & $\mathrm{NI}$ \\
D-Glucose & $\mathrm{NI}$ & $\mathrm{NI}$ \\
D-Maltose & $\mathrm{NI}$ & $\mathrm{NI}$ \\
\hline
\end{tabular}

$\S$ Concentration, $\mu \mathrm{g} / \mathrm{mL}$, NI, the absence of inhibition at $500 \mathrm{mM}$.

(A)

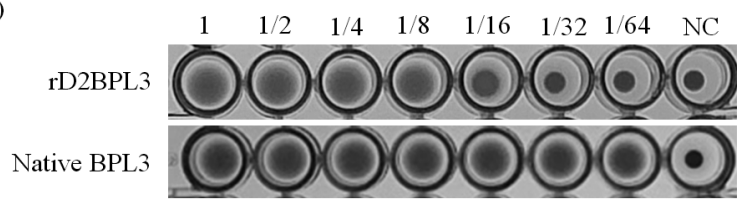

(B)
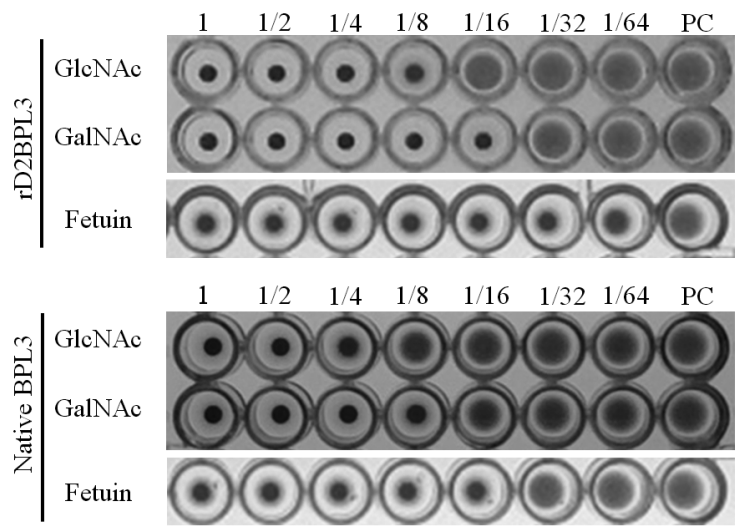

Figure 6. Hemagglutination activity and inhibition of rD2BPL3 (A) Hemagglutination activity of rD2BPL3 $(100 \mu \mathrm{g} / \mathrm{mL})$ and native BPL3 $(50 \mu \mathrm{g} / \mathrm{mL})$; A serial twofold dilution was obtained (left to right); (B) Inhibition test of rD2BPL3 $(25 \mu \mathrm{g} / \mathrm{mL})$ and native BPL3 $(1.5 \mu \mathrm{g} / \mathrm{mL})$; NC, negative control; PC, positive control.

\subsection{Glycan Micro-Array Analysis}

We used a glycoconjugate microarray to determine the glycan binding properties of rD2BPL3, rD1BPL3, and native BPL3. rD1BPL3 did not bind to any sugars on the Glycan-100 array (Figure 7). rD2BPL3 and native BPL3 exhibited partially different glycan binding specificities, except for the monosaccharide $\alpha$-Man-Sp (over RFU, Relative Fluorescence Units, 1000), Gal- $\alpha-1,3-G a l-\beta-1,3-$ GlcNAc- $\beta$-Sp and Gal- $\alpha-1,4-$ Gal- $\beta-1,3-$ GlcNAc- $\beta$-Sp (Figure 7, Table 2). Interestingly, native BPL3 did not bind to GlcNAc- $\beta$-Sp or GalNAc- $\beta$-Sp; these mono-saccharides were able to inhibit hemagglutination activity. rD2BPL3 bound to GlcNAc- $\beta$-Sp. Recombinant lectin had specificity to the beta ( $\beta$-) conformation, e.g., Gal- $\beta-1,3-G l c N A c-\beta-S p$, LacdiNAc (GalNAc- $\beta-1,4-G l c N A c-\beta-S p 2)$, and GlcNAc- $\beta-1,2-M a n-\alpha-S p$. Native BPL3 exhibited specificity to the alpha $(\alpha-)$ conformation, e.g., Gal- $\beta-1,3-G a l N A c-\alpha-S p$ (T-antigen, core structure type 3), GlcNAc- $\beta-1,6-($ Gal- $\beta-1,3)-G a l N A c-\alpha-O-S e r-S p 4$, and Neu5Ac- $\alpha-2,6-$ Gal- $\beta-1,3-($ Neu5Ac- $\alpha-2,6)$-GalNAc- $\beta$-Sp (Figure 7, Table 2). 


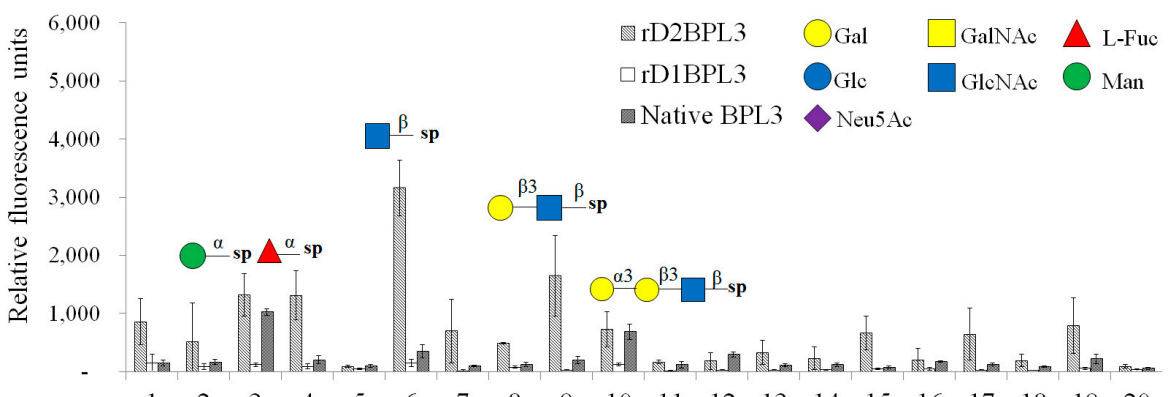

$\begin{array}{llllllllllllllllllll}1 & 2 & 3 & 4 & 5 & 6 & 7 & 8 & 9 & 10 & 11 & 12 & 13 & 14 & 15 & 16 & 17 & 18 & 19 & 20\end{array}$

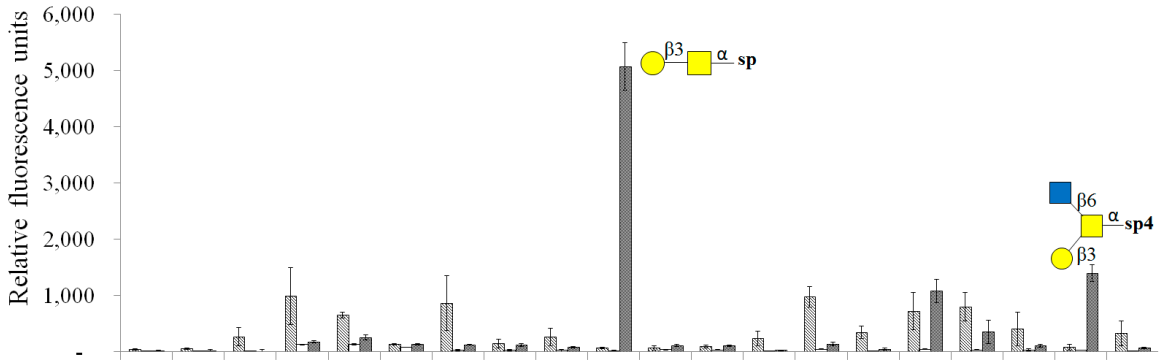

$\begin{array}{llllllllllllllllllll}21 & 22 & 23 & 24 & 25 & 26 & 27 & 28 & 29 & 30 & 31 & 32 & 33 & 34 & 35 & 36 & 37 & 38 & 39 & 40\end{array}$
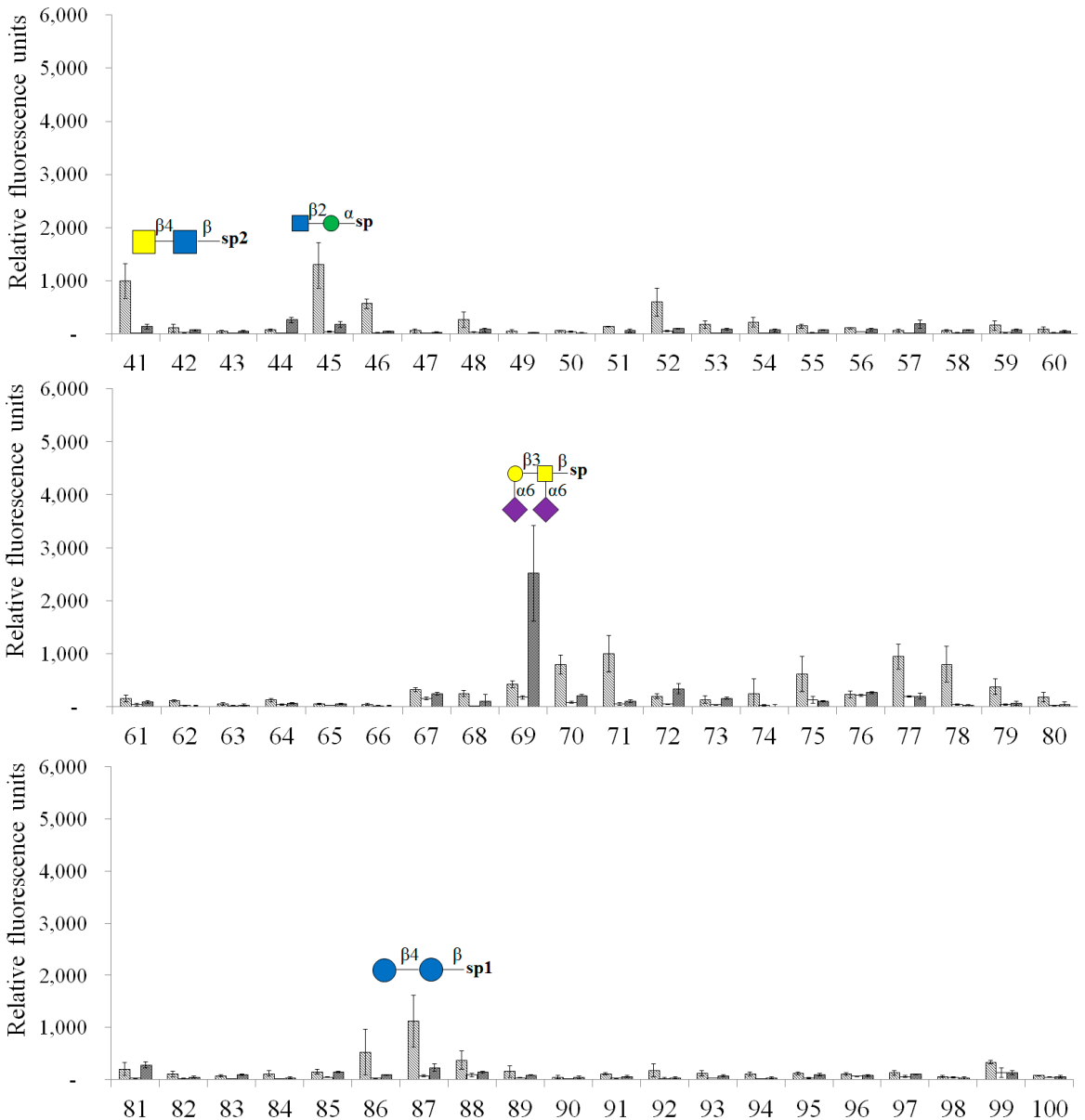

Figure 7. Glycan array of recombinant and native BPL3. Relative fluorescence units were calculated using an array analysis program (RayBioTech). The signal exceeding 1000 units is marked with the glycan structure. 
Table 2. Overview of carbohydrate structures recognized by rD2BPL3 and native BPL3.

\begin{tabular}{|c|c|c|c|}
\hline \multirow{2}{*}{ No. } & \multirow{2}{*}{ Glycan Structure } & \multicolumn{2}{|c|}{ RFU (Normalized) } \\
\hline & & rD2BPL3 & Native BPL3 \\
\hline \multicolumn{4}{|c|}{ Monosaccharides } \\
\hline 3 & $\alpha$-Man-Sp & 1322 & 1025 \\
\hline 4 & $\alpha$-Fuc-Sp & 1313 & 205 \\
\hline 6 & $\beta$-GlcNAc-Sp & 3164 & 348 \\
\hline \multicolumn{4}{|c|}{ Disaccharides } \\
\hline 9 & Gal- $\beta-1,3-G l c N A c-\beta-S p$ & 1646 & 201 \\
\hline 30 & Gal- $\beta-1,3-G a l N A c-\alpha-S p$ & 64 & 5075 \\
\hline 41 & GalNAc- $\beta-1,4-G l c N A c-\beta-S p 2$ & 993 & 134 \\
\hline 45 & GlcNAc- $\beta-1,2-$ Man- $\alpha-S p$ & 1290 & 173 \\
\hline 87 & D-Cellose- $\beta$-Sp1 & 1119 & 225 \\
\hline \multicolumn{4}{|c|}{ Gangliosides and Sialylated Oligosaccharides } \\
\hline 24 & Neu5Ac- $\alpha-2,6$-Gal- $\beta-1,4-$-Glc- $\beta$-Sp & 991 & 178 \\
\hline 69 & Neu5Ac- $\alpha-2,6-$ Gal- $\beta-1,3-($ Neu5Ac- $\alpha-2,6)$-GalNAc- $\beta$-Sp & 421 & 2518 \\
\hline 71 & Neu5Ac- $\alpha-2,6-($ Neu 5 Ac- $\alpha-2,3)-G a l-\beta-1,3-G a l N A c-\beta-S p$ & 993 & 96 \\
\hline \multicolumn{4}{|c|}{ Blood Groups, Lewis Antigens and Fucosylated Oligosaccharides } \\
\hline 34 & Neu5Ac- $\alpha-2,3-$-Gal- $\beta-1,3-($ Fuc- $\alpha-1,4)$-GlcNAc- $\beta$-[Sialyl Lewis A]-Sp & 973 & 138 \\
\hline \multicolumn{4}{|c|}{ Globo series, Milk Oligosaccharides and GAGs } \\
\hline 36 & Gal- $\alpha-1,4-G a l-\beta-1,3-G l c N A c-\beta-S p$ & 721 & 1082 \\
\hline \multicolumn{4}{|c|}{ O-Glycan, $N$-Glycans and $\alpha$-Gal } \\
\hline 39 & GlcNAc- $\beta-1,6-($ Gal- $\beta-1,3)-$ GalNAc- $\alpha-O-S e r-S p 4$ & 77 & 1398 \\
\hline \multicolumn{4}{|c|}{ Natural Oligosaccharides } \\
\hline 77 & Glc- $\alpha-1,6-G l c-\alpha-1,4-G l c-\beta-S p 1$ & 941 & 190 \\
\hline
\end{tabular}

\section{Discussion}

In the past few decades, various Bryopsis plumosa lectins (Bryohealin, BPL2-4) have been purified $[12,13,20-22]$, but their biochemical properties are unclear, owing to the lack of a sufficient amount of active recombinant protein for analyses. In particular, the high sequence similarity between BPL3 and BPL4 (60\%) and similar molecular properties have limited their applications $[12,13]$. Thus, recombinant protein production was necessary.

Monomeric (rD1BPL3) and dimeric (rD2BPL3) proteins were designed based on native BPL3 cDNA and expressed in a bacterial expression system. rD2BPL3 was highly expressed, while the monomeric recombinant showed weak expression or no expression. The bacterial expression system was a sufficient substitute for lectin production from native sources. The expression efficiency of rD2BPL3 was affected by temperature, but IPTG concentration had minimal effects. Approximately $3 \mathrm{mg}$ of rD2BPL3 was produced from $1 \mathrm{~L}$ bacterial culture, while the yield of BPL3 was $0.017 \mathrm{mg} / \mathrm{g}$ of Bryopsis plumosa (data not shown) [12]. Although the productivity was lower than that reported for other recombinant lectins (16.5 mg/L for rRhodobindin [14], $16 \mathrm{mg} / \mathrm{L}$ for frutalin [23], and $15 \mathrm{mg} / \mathrm{L}$ for Microcystis viridis lectin [24]), it is an acceptable value when considering the yield of native BPL3 and other native lectins (0.1 mg/L for Glycin max lectin [25], 2-5 mg for Pisum sativum lectin [26], and $5 \mathrm{mg} / \mathrm{L}$ Allium sativum [27]).

Recombinant lectin production is an efficient way to overcome obstacles to the application of lectins derived from natural sources because it guarantees a substantial supply of pure lectins for biomedical applications [28]. Despite their advantages, prokaryotic expression systems have issues with respect to the creation of a proper lectin structure, leading to the frequent production of a biologically inactive protein [14]. Eukaryotic expression systems overcome the limitations of prokaryotic expression systems owing to their post-translational modification ability (e.g., the production of glycosylated proteins). Considering disadvantages with respect to genetic transformation efficiency, the efficiency of expression, immune responses, and the cost of culture [24,29], bacterial expression systems are still widely used for the production of active proteins. 
In this study, recombinant BPL3 was successfully produced using an artificially constructed tandem repeat structure in E. coli. Although tandem repeat domain structures have been detected in native lectins, such as the Rhamnose-binding lectin from Silurus asotus eggs [17], Galectin from Caenorhabditis elegans [30], and mannose-binding lectin from Boodlea coacta [18], the contribution of tandem repeat structure to recombinant protein production is still unclear.

Previously, Han et al. found that the red algal lectin Rhodobindin consists of a heterologous tandem repeat sequence, and this structure may contribute to protein solubility and hemagglutination activity [14]. The tetrameric structure is advantageous relative to the dimeric form, with 15-30 times greater activity levels for the same concentration [14]. Mixing 2 dimeric domains did not affect their activity; in fact, the activity was enhanced by the formation of the tetrameric structure. Therefore, the construction of a tandem repeat structure is a potential tool for the production of recombinant lectin $[14,31]$. The expression efficiency of rD2BPL3 was enhanced and hemagglutination activity was 15 times weaker than that of native BPL3. Therefore, we assume that the hemagglutination activity can be improved by construction of trimeric or tetrameric forms, as reported in the previous study [14]. These results support the hypothesis that the tandem repeat structure facilitates lectin production in E. coli.

Although an enhancement in lectin solubility was predicted in a previous study, rD2BPL3 could not be solubilized in a common buffer system, PBS. Most lectins contain at least two domains that interact by dimerization or multimerization e.g., [32]. The peptide sequence of BPL3 exhibited similarity to an $\mathrm{H}$ type lectin, Helix pomatia Agglutinin (HPA), produced by invertebrates [12]. HPA contains two trimeric peptides linked by intramolecular disulfide bonds [33]. Trimerization of HPA occurs by a strong hydrophobic cluster with amino acids in the $\mathrm{N}$ - and $\mathrm{C}$-terminal regions of the neighboring monomer (Supplementary Figure S4). rD2BPL3 designed with two identical domains is probably insufficient to generate hydrophobic interactions between domains, and the formation of inclusion bodies resulted. We assumed that enhanced solubility and activity may be possible by constructing a trimeric repeat structure (Supplementary Figure S4). rD2BPL3 was solubilized in denaturing conditions and refolded by flash dilution methods.

rD2BPL3 showed similar sugar specificity to that of native BPL3. rD1BPL3 was unable to agglutinate human blood cell in this study, but this result needs further validation considering the fact that experiments involving rD1BPL3 could not be replicated satisfactorily because it frequently failed to be expressed. Hemagglutination activity was inhibited by treatment with a complementary sugar, D-GlcNAc or D-GalNAc, used at half the concentrations required to inhibit the activity of rD2BPL3. The concentrations of the complimentary sugars were determined based on the assumption that the affinity of rD2BPL3 for the sugars may be less than that of native BPL3 due to misfolding or the presence of urea in the buffer, which can break ionic bonds and induce a hydrophobic effect [34]. Recombinant acylpeptide hydrolase displayed $15 \%$ of its original activity at $8 \mathrm{M}$ urea. It has been reported that protein secondary structure is destroyed at urea concentrations of $2 \mathrm{M}$ and higher [35].

Moreover, biochemical properties, such as the divalent ion requirement and heat stability, were undistinguishable from those of native BPL3. These results can be explained by the similarity in protein structure between rD2BPL3 and native BPL3.

A glycan array is a powerful tool for functional glycomics [36]. We used a glycan array to compare glycan recognition properties between native and recombinant proteins. In contrast to the inhibition test results, the array results indicated that rD2BPL3 and native BPL3 differed with respect to glycan binding properties on the anomeric center of glycan and amino-glycan. Native BPL3 could not bind to $\beta$-GlcNAc and $\beta$-GalNAc, but the recombinant exhibited strong binding to $\beta$-GlcNAc. Native BPL3 showed a preference for the alpha $(\alpha-)$ conformation of amino sugars, and rD2BPL3 exhibited binding to the beta $(\beta-)$ conformation of amino sugars, such as LacdiNAc. The carbohydrates, $\alpha$-GalNAc, $\beta$-GlcNAc and $\beta$-GalNAc, exhibit only slight structural differences. The molecules are designated $\alpha-$ and $\beta$ - based on the orientation of the hydroxyl group at carbon $1(\mathrm{C} 1)$, while the designations Gal and Glc are based on the orientation of the hydroxyl group at carbon 4 (C4). In nature, these sugars mostly 
occur as a mixed form of amino sugars (alpha $(\alpha-)$ and beta ( $\beta$-) conformation). Thus, treatment with these sugars could inhibit the agglutination of human erythrocytes.

The distance between $\mathrm{C} 1$ of GlcNAc and candidate binding site amino acids in artificially constructed rD2BPL3 - ${ }^{78}$ aspartic acid (D78) and ${ }^{35}$ Serine(S35) - was shorter than that between native BPL3 (Supplementary Figure S4). Dimerization likely causes the BPL3 structure to be tighter than that of rD1BPL3 or native BPL3, and this could explain the binding differences between the two forms. The presence of urea in the buffer could be another reason for these differences. The secondary structure of recombinant acylpeptide hydrolase was destroyed in a system containing urea, resulting in loss of enzyme activity [35]. It is difficult to investigate the difference in sugar specificity without structural analysis. Therefore, crystallographic analysis might be needed for deeper understanding of the binding differences. Recombinant and native lectins do not show identical properties. In a comparative analysis, recombinant and native frutalin exhibited different binding properties in prostate tissues [37]. The distinct carbohydrate-binding affinities of the two forms explain this difference in binding $[37,38]$.

Glycosylation and post-translational modifications were not important factors, as evidenced by the lack of post-translational modifications of native BPL3 in a mass spectrometry analysis [12]. The recombinants were consistent with lectins reported in previous studies. In fact, BPL3 was isolated from a mixture of lectins (Bryohealin) based on a competitive binding assay according to sugar specificity (GlcNAc >> GalNAc) [12].

Native BPL3 may consist of 2-6 domains with hydrophobic interactions. BPL3 lacked free sulfhydryl groups; thus, interactions between domains may be flexible. The recombinant protein contained a peptide linker ( 4 amino acids) to connect homologous domains, resulting in potential tension in the structure. Recombinant WFA with a C272 mutation showed limited binding specificity to GalNAc-terminated glycans [39]; thus, the cysteine residue is important for the maintenance of activity. BPL3 had two intramolecularly connected cysteines. Using the bacterial recombinant system, disulfide bond formation was difficult. It could be concluded that the formation of disulfide bonds is an important determinant of sugar specificity.

The purity of native BPL3 was not high, and a BPL4 band was detected in the SDS-PAGE results (Supplementary Figure S3). In addition, sequences encoding more than four peptides similar to BPL3 were found in the Bryopsis genome (unpublished, data not shown, cut-off value: e-50). A mixture of lectins could interact mutually, resulting in structural changes that may influence carbohydrate specificity. The effect of denaturants on protein structure is another candidate hypothesis. The denaturant may affect the protein conformation. In a solution with a denaturant, alterations in protein structure and the solvent structure around the protein are possible [40]. However, the precise mechanisms underlying differences in sugar specificity between native and recombinant proteins are unclear; thus, more intensive studies of protein structure are needed.

HPA is predicted to protect fertilized eggs from bacteria and is part of the innate immune system of the snail [33]. Several sea slugs that are closely related to the snail do not have any such lectins, but symbiotic algae, Bryopsis spp., produce similar lectins. We predict that Bryopsis lectins have two roles, i.e., protection from mechanical damage and from bacteria in the vicinity of damaged cells and protection of fertilized eggs of symbiotic sea slugs from bacteria; protoplast formation via mechanical damage by sea slugs is a reproduction strategy [41]. BPL3 has potential as an anti-microbial reagent.

Diverse applications of lectins or sugar-binding proteins have been reported [11,42]. High mannose-binding lectin has received attention owing to its potential to inhibit HIV-1 and influenza virus [18]. Lectins have been identified as simple and convenient for histochemical analyses or as biomarkers for disease detection. HPA (with specificity to $\alpha$-GalNAc, $\alpha$-GlcNAc, and $\alpha$-Gal) has been studied in the normal human prostate, benign prostatic hyperplasia, and prostatic carcinoma [43]. WFA has been used as a tool for revealing areal borders and subdivisions. As LacdiNAc $(\beta$-D-GalNAc-[1 $\rightarrow 4$ ]-D-GlcNAc) is associated with tumor malignancy in leukemia, prostate, pancreatic, ovarian, and liver cancers, WFA is promising for cancer glycobiomarker detection [10,39]. rD2BPL3 
recognizes LacdiNAc, similarly to WFA; thus, rD2BPL3 is strong candidate for the development of a cancer glycobiomarker.

In our study, recombinant BPL3 was successfully produced using an artificially constructed tandem repeat structure and it demonstrated various advantages for the preparation of pure lectin for industrial purposes. This method may be useful for the production of active proteins. Both lectins (native and recombinant) could be useful histochemical biomarkers.

\section{Materials and Methods}

\subsection{Preparation of Native BPL3}

Native BPL3 was prepared according to the methods of Han et al. [12]. Bryopsis plumosa was ground to a fine powder in liquid nitrogen and dissolved in 5 volumes of $1 \times$ phosphate-buffered saline (PBS). Cell debris was removed by centrifugation and the supernatant was collected as a crude extract. The supernatant was directly evaluated by GalNAc-agarose chromatography and BPL3 was eluted by adding $0.2 \mathrm{M}$ GlcNAc in $1 \times$ PBS. The purified protein was confirmed by SDS-PAGE. Fractions with active proteins were pooled and dialyzed in $1 \times$ PBS overnight.

\subsection{Cloning and Construction of the Recombinant Protein}

The BPL3 cDNA sequence was obtained from the NCBI database (Accession number KX867966). The cDNA was codon-optimized to bacteria K-12 using Geneious ver. 8.1. cDNA was synthesized to mimic the protein with a two-homologous domain fusion structure (rD2BPL3) with restriction enzyme sites from Bioneer (Deajeon, Korea) and cloned into the PBHA vector. BPL3 DNA was digested and cloned into pET28a (+) (Invitrogen, Carlsbad, CA, USA) (Figure 1). The synthesized BPL3 and pET28a $(+)$ were digested with two enzymes, BamHI and SacI (monomeric, rD1BPL3) or BamHI and HindIII (dimeric, rD2BPL3), at $37^{\circ} \mathrm{C}$ for $2 \mathrm{~h}$. After digestion, the DNA was purified using the Qiagen Gel Extraction Kit (Valencia, CA, USA). The purified DNA was cloned into pET28a (+) by incubation at $12{ }^{\circ} \mathrm{C}$ overnight with 4 units of T4 DNA ligase. The plasmid was transformed into the cloning host DH5 $\alpha$ and spread on LB agar plates containing $25 \mu \mathrm{g} / \mathrm{mL}$ kanamycin. The positive colonies were isolated and sub-cultured in $10 \mathrm{~mL}$ of LB-kanamycin medium. The plasmid containing the BPL3 sequence was purified and stored at $-20^{\circ} \mathrm{C}$ until use.

pET28a::BPL3 was transformed into the expression hosts BL21(XDE3), BL21(DE3)pLysS, BL21(DE3) codon-plus RIL, and Rosetta(DE3) (Invitrogen, Carlsbad, CA, USA) to determine the optimal host system. The transformants were spread on LB agar plates containing $25 \mu \mathrm{g} / \mathrm{mL}$ kanamycin. The positive colonies were isolated and sub-cultured in $10 \mathrm{~mL}$ of LB-kanamycin medium.

\subsection{Optimization of rBPL3 Expression}

The transformants were inoculated in LB medium containing kanamycin $(25 \mu \mathrm{g} / \mathrm{mL})$ and cultured overnight at $37^{\circ} \mathrm{C}$. The subculture was diluted 1:100 in $100 \mathrm{~mL}$ of LB medium and grown for $1-4 \mathrm{~h}$ at $37^{\circ} \mathrm{C}$ in an Erlenmeyer flask in a shaker until reaching OD 0.4-0.6. When the designated OD was reached, $1 \mathrm{~mL}$ of sample was removed from the flask and the cell pellet was collected by centrifugation (un-induced control). To induce the protein, IPTG (final concentration, $0.4 \mathrm{mM}$ ) was added and cultured at various temperatures $\left(20^{\circ} \mathrm{C}, 25^{\circ} \mathrm{C}, 30^{\circ} \mathrm{C}\right.$, and $\left.37^{\circ} \mathrm{C}\right)$ overnight. To determine the optimum concentration of IPTG, various concentrations of IPTG $(0.1,0.2,0.4$, and $1 \mathrm{mM})$ were added to the bacterial culture (OD $0.4-0.6)$ overnight. The effects of various incubation times $(1,3$, and $5 \mathrm{~h}$ and overnight) were determined by incubating bacteria after the addition of IPTG $(0.4 \mathrm{mM})$. Aliquots $(5 \mathrm{~mL})$ were collected after incubation in different conditions.

Total proteins from each cell culture were extracted and analyzed to determine the expression levels of rD1BPL3 and rD2BPL3. Total protein extracts were prepared by heating samples for $5 \mathrm{~min}$ at $90^{\circ} \mathrm{C}$ after treatment in $1 \times$ SDS-PAGE sample buffer $(0.2 \mathrm{~mL} / \mathrm{mL}$ culture $)$ to the cell precipitates. The extracts were centrifuged at $20,000 \times g$ and the supernatants were used directly for SDS-PAGE. 
Soluble fractions were obtained from cell precipitates after cell culture $(5 \mathrm{~mL})$. Resuspended samples in $1 \mathrm{~mL}$ of extraction buffer (1× PBS, $10 \mathrm{mM}$ imidazole, $1 \mathrm{mM}$ PMSF, pH 7.2) were sonicated at a $15 \%$ amplitude, repeated 20 times, with 3-second on/off periods. Supernatants were collected by centrifugation at $20,000 \times g$ for $10 \mathrm{~min}$. The expression efficiency was determined by calculating the target band intensity after SDS-PAGE using GelAnalyzer 2010 (http:/ / www.gelanalyzer.com/). 4.4. Purification of rBPL3.

\subsection{Purification of rBPL3.}

The bacterial cell culture $(500 \mathrm{~mL})$ was centrifuged at $5000 \times g$ for $10 \mathrm{~min}$ and resuspended in $50 \mathrm{~mL}$ of urea extraction buffer $\left(50 \mathrm{mM} \mathrm{NaH}_{2} \mathrm{PO}_{4}, 300 \mathrm{mM} \mathrm{NaCl}, 8 \mathrm{M}\right.$ urea, pH8.0). Suspensions were sonicated at a 15\% amplitude, repeated 20 times with 3-second on/off periods, and centrifuged at $20,000 \times g$ for $10 \mathrm{~min}$ to pellet the cellular debris. The supernatant was collected as a crude extract. The bacterial extract ( $50 \mathrm{~mL}$ ) was directly added to $1 \mathrm{~mL}$ Ni-NTA chromatography columns (Qiagen) using the FPLC chromatography system (Bio-Rad, Richmond, CA, USA) with a $1 \mathrm{~mL} / \mathrm{min}$ flow-rate. The column was washed with 15 volumes of wash buffer, $50 \mathrm{mM} \mathrm{NaH} \mathrm{PO}_{4}, 300 \mathrm{mM} \mathrm{NaCl}, 8 \mathrm{M}$ urea, $25 \mathrm{mM}$ imidazole, pH 8.0. Recombinant BPL3 was eluted with an imidazole step gradient, 5 volumes of $75 \mathrm{mM}, 125 \mathrm{mM}$, and $250 \mathrm{mM}$ imidazole in extraction buffer. Fractions were collected and analyzed by SDS-PAGE. Fractions including the pure protein were pooled.

\subsection{Refolding of $r B P L 3$}

rD2BPL3 inclusion bodies were refolded by the flash dilution method [44]. Purified denatured rD2BPL3 was filtered using a $0.45-\mu \mathrm{m}$ syringe filter and quickly added to $2,4,8,16$, and 40 volumes of refolding buffer $(1 \times$ PBS with $0.3 \mathrm{M} \mathrm{NaCl}, \mathrm{pH} 7.5)$. The final concentration of protein was approximately $5-100 \mu \mathrm{g} / \mathrm{mL}$. The diluted sample was incubated at $20^{\circ} \mathrm{C}$ for $3 \mathrm{~h}$. The insoluble material was removed by centrifugation at $20,000 \times \mathrm{g}$ for $10 \mathrm{~min}$ at room temperature and the supernatant was collected as refolded rD2BPL3. For quantifying the soluble fractions, diluted samples were re-concentrated to original volume using speed-vac evaporator (Labcono, MO, USA). Same volume of sample was subjected to SDS-PAGE, and the soluble fraction was quantified based on protein band intensity.

\subsection{Hemagglutination Activity Assay}

Hemagglutination activity was tested following the protocols described by Han et al. [12]. Human blood type B was obtained from a healthy donor and washed with PBS. A serial two-fold dilution of the purified rD2BPL3 was made in a final volume of $25 \mu \mathrm{L}$ of PBS in 96-well microtiter plates, and $25 \mu \mathrm{L}$ of erythrocyte suspension (4\%) was added to each well. The minimum amount of lectin needed for agglutination was defined as 1 hemagglutinating unit (HU).

\subsection{Determination of Carbohydrate Specificity}

Carbohydrate specificity was determined by inhibition test of hemagglutination activity and a glycan micro-array.

For the inhibition test, mono- and disaccharides at $500 \mathrm{mM}$ or $100 \mathrm{mg} / \mathrm{mL}$ glycoprotein were used as inhibitors of lectin: $N$-acetyl-glucosamine, $N$-acetyl-galactosamine, L-fucose, D-galactose, D-glucose, D-mannose, D-fructose, $\beta$-lactose, D-maltose, and fetuin. Serial two-fold dilutions of sugar samples were prepared in PBS and mixed with an equal volume of 4 HU rD2BPL3 and native BPL3. An equal volume $(25 \mu \mathrm{L})$ of a $4 \%$ human erythrocyte suspension was added to the sugar-lectin mixture. The minimum inhibitory concentration of the sugar in the final reaction mixture was calculated. 


\subsection{Glycan Microarray}

A glycan microarray analysis was performed by Ebiogen (Seoul, Korea). The Glycan Array kit was purchased from RayBioTech (Norcross, GA, USA). An array containing 100 synthetic glycans printed in quadruplicate on a glass slide was used. Label-based detection was performed according to the manufacturer's protocols. Biotinylated recombinant lectins and native lectins at $50 \mu \mathrm{g} / \mathrm{mL}$ were added to array wells and incubated for $>3 \mathrm{~h}$ with gentle rocking. The glass slide was washed with $1 \times$ wash buffer I and II, provided in the kit. Glycan-lectin binding was detected by incubation with Cy3 equivalent dye-conjugated streptavidin for $1 \mathrm{~h}$ at room temperature. For cyanine-3 detection, the signals were visualized using a microarray laser scanner (Genfix 4100A; Molecular Devices, Sunnyvale, CA, USA) with excitation at $554 \mathrm{~nm}$ and emission at $568 \mathrm{~nm}$. Data extraction was performed using the microarray analysis software Genfix. Glycan array data were normalized and analyzed using RayBio Analysis software (GA-Glycan-100-SW, RayBioTech).

\subsection{Effect of Temperature and Divalent Cations on the Agglutination Activity}

Heat stability was examined according to the methods of Han et al. [13]. Heated aliquots of purified rD2BPL3 were prepared by incubation at various temperatures $\left(4-100{ }^{\circ} \mathrm{C}\right)$ for $30 \mathrm{~min}$. The samples were cooled to room temperature and insoluble materials were removed by centrifugation at $12,000 \times g$ for $1 \mathrm{~min}$. The results were expressed as the relative hemagglutination activity shown by the heated samples compared to the non-heated sample (control), representing 100\%. The effect of divalent metal ions was determined by adding EDTA or $\mathrm{CaCl}_{2}$ at $5 \mathrm{mM}$ in the protein solution.

\subsection{Mass Spectrometry}

Protein bands obtained by SDS-PAGE were excised, in-gel digested with trypsin, and cleaned with Zip-Tip (Millipore, Billerica, MA, USA). Mass analyses were performed using Capillary LC-Nano ESI-MS with a 6545 Q-TOF LC/MS (Agilent Technologies, Santa Clara, CA, USA). Samples were applied to a ZORBAX 300SB-C8 column $(1 \times 50 \mathrm{~mm}, 3.5 \mu \mathrm{m}$; Agilent $)$ equilibrated with $0.1 \%(v / v)$ formic acid in mass grade water and eluted by a gradient between water and $100 \%$ acetonitrile at a flow rate of $10 \mu \mathrm{L} / \mathrm{min}$. The tuning parameters used for mass analyses were as follows: capillary temperature $300{ }^{\circ} \mathrm{C}$, source voltage $1.9 \mathrm{kV}$, skimmer voltage $45 \mathrm{~V}$, and fragmentor voltage $175 \mathrm{~V}$.

\section{Conclusions}

Recombinant BPL3 from the coenocytic marine green alga B. plumosa was developed using an E. coli expression system combined with an artificially constructed tandem repeat structure. The repeat domain contributed to the high expression of the active protein. The recombinant protein recognized $N$-acetyl- $\beta$-D-glucosamine, Gal- $\beta-1,3-G l c N A c$, LacdiNAc, and GlcNAc- $\beta-1,2-M a n$. The process developed in this study was suitable for the quality-controlled production of high amounts of soluble recombinant lectins. These results indicate that both lectins (native and recombinant) may have applications as histochemical biomarkers for cancer.

Supplementary Materials: The following are available online at www.mdpi.com/1660-3397/16/1/13/s1, Supplementary Figure S1: Expression efficiency of rD2BPL3 for various expression hosts, Supplementary Figure S2: Confirmation of the peptide sequence using LC-MS/MS, Supplementary Figure S3: Purification of native BPL3, Supplementary Figure S4: Predicted 3-dimensional structure of BPL3, Supplementary Table S1: Glycan array of recombinant and native BPL3. Binding signals were normalized using a program provided by RayBioTech.

Acknowledgments: This work was supported by a grant from MABIK (2017M01300, MABIK program). We would like to thank Editage (www.editage.co.kr) for English language editing.

Author Contributions: Gwang Hoon Kim and Jong Won Han conceived and designed the experiments. Jong Won Han, Hyun-Ju Hwang, and Jin-Woo Han performed the experiments. Gwang Hoon Kim and Jong Won Han analyzed the data. Jong Won Han contributed reagents/materials/analysis tools. Gwang Hoon Kim, Jong Won Han, and Hyun-Ju Hwang wrote the paper.

Conflicts of Interest: The authors declare no conflict of interest. 


\section{References}

1. Dan, X.; Liu, W.; Ng, T.B. Development and applications of lectins as biological tools in biomedical research. Med. Res. Rev. 2016, 36, 221-247. [CrossRef] [PubMed]

2. Zheng, T.; Peelen, D.; Smith, L.M. Lectin arrays for profiling cell surface carbohydrate expression. J. Am. Chem. Soc. 2005, 127, 9982-9983. [CrossRef] [PubMed]

3. Norton, P.; Comunale, M.A.; Herrera, H.; Wang, M.; Houser, J.; Wimmerova, M.; Romano, P.R.; Mehta, A. Development and application of a novel recombinant Aleuria aurantia lectin with enhanced core fucose binding for identification of glycoprotein biomarkers of hepatocellular carcinoma. Proteomics 2016, 16, 3126-3136. [CrossRef] [PubMed]

4. Strathmann, M.; Wingender, J.; Flemming, H.C. Application of fluorescently labelled lectins for the visualization and biochemical characterization of polysaccharides in biofilms of Pseudomonas aeruginosa. J. Microbiol. Methods 2002, 50, 237-248. [CrossRef]

5. Hortin, G.L.; Trimpe, B.L. Lectin affinity chromatography of proteins bearing O-linked oligosaccharides: Application of jacalin-Sepharose. Anal. Biochem. 1990, 188, 271-277. [CrossRef]

6. Hovhannisyan, V.A.; Bazukyan, I.L.; Gasparyan, V.K. Application of silver nanoparticles and CdSe quantum dots sensitized with of C-like lectin for detection of St. aureus. Comparison of various approaches. Talanta 2017, 175, 366-369. [CrossRef] [PubMed]

7. Seitz, A.; Colodel, E.; Schmitz, M.; Gimeno, E.; Driemeier, D. Use of lectin histochemistry to diagnose Sida carpinifolia (Malvaceae) poisoning in sheep. Vet. Rec. 2005, 156, 386-388. [CrossRef] [PubMed]

8. Hsu, K.L.; Gildersleeve, J.C.; Mahal, L.K. A simple strategy for the creation of a recombinant lectin microarray. Mol. Biosyst. 2008, 4, 654-662. [CrossRef] [PubMed]

9. Singh, R.S.; Walia, A.K.; Kanwar, J.R.; Kennedy, J.F. Amoebiasis vaccine development: A snapshot on E. histolytica with emphasis on perspectives of Gal/GalNAc lectin. Int. J. Biol. Macromol. 2016, 91, 258-268. [CrossRef] [PubMed]

10. Haji-Ghassemi, O.; Gilbert, M.; Spence, J.; Schur, M.J.; Parker, M.J.; Jenkins, M.L.; Burke, J.E.; van Faassen, H.; Young, N.M.; Evans, S.V. Molecular basis for recognition of the cancer glycobiomarker, LacdiNAc (GalNAc[beta1 $\rightarrow 4$ ]GlcNAc), by Wisteria floribunda Agglutinin. J. Biol. Chem. 2016, 291, 24085-24095. [CrossRef] [PubMed]

11. Teixeira, E.H.; Arruda, F.V.S.; Nascimento, K.S.; Carneiro, V.A.; Nagano, C.S.; Silva, B.R.; Sampaio, A.H.; Cavada, B.S. Biological applications of plants and algae lectins: An overview. In Carbohydrates-Comprehensive Studies on Glycobiology and Glycotechnology; InTech: Rijeka, Croatia, 2012; Chapter 23; pp. 533-558.

12. Han, J.W.; Yoon, K.S.; Klochkova, T.A.; Hwang, M.S.; Kim, G.H. Purification and characterization of a lectin, BPL-3, from the marine green alga Bryopsis plumosa. J. Appl. Phycol. 2011, 23, 745-753. [CrossRef]

13. Han, J.W.; Yoon, K.S.; Jung, M.G.; Chah, K.H.; Kim, G.H. Molecular characterization of a lectin, BPL-4, from the marine green alga Bryopsis plumosa (Chlorophyta). Algae 2012, 27, 55-62. [CrossRef]

14. Han, J.W.; Jung, M.G.; Shim, E.Y.; Shim, J.B.; Kim, Y.M.; Kim, G.H. Functional recombinants designed from a fetuin/asialofetuin-specific marine algal lectin, rhodobindin. Mar. Drugs 2015, 13, 2183-2195. [CrossRef] [PubMed]

15. Lam, S.K.; Ng, T.B. Lectins: Production and practical applications. Appl. Microbiol. Biotechnol. 2011, 89, 45-55. [CrossRef] [PubMed]

16. Oliveira, C.; Teixeira, J.A.; Domingues, L. Recombinant production of plant lectins in microbial systems for biomedical application-The frutalin case study. Front. Plant Sci. 2014, 5. [CrossRef] [PubMed]

17. Hosono, M.; Ishikawa, K.; Mineki, R.; Murayama, K.; Numata, C.; Ogawa, Y.; Takayanagi, Y.; Nitta, K. Tandem repeat structure of rhamnose-binding lectin from catfish (Silurus asotus) eggs. Biochim. Biophys. Acta 1999, 1472, 668-675. [CrossRef]

18. Sato, Y.; Hirayama, M.; Morimoto, K.; Yamamoto, N.; Okuyama, S.; Hori, K. High mannose-binding lectin with preference for the cluster of alpha1-2-mannose from the green alga Boodlea coacta is a potent entry inhibitor of HIV-1 and influenza viruses. J. Biol. Chem. 2011, 286, 19446-19458. [CrossRef] [PubMed]

19. Shim, E.; Shim, J.; Klochkova, T.A.; Han, J.W.; Kim, G.H. Purification of a sex-specific lectin involved in gamete binding of Aglaothamnion callophyllidicola (Rhodophyta). J. Phycol. 2012, 48, 916-924. [CrossRef] [PubMed] 
20. Han, J.W.; Jung, M.G.; Kim, M.J.; Yoon, K.S.; Lee, K.P.; Kim, G.H. Purification and characterization of a D-mannose specific lectin from the green marine alga, Bryopsis plumosa. Phycol. Res. 2010, 58, 143-150. [CrossRef]

21. Yoon, K.S.; Lee, K.P.; Klochkova, T.A.; Kim, G.H. Molecular characterization of the lectin, Bryohealin, involved in protoplast regeneration of the marine alga Bryopsis plumosa (Chlorophyta). J. Phycol. 2008, 44, 103-112. [CrossRef] [PubMed]

22. Jung, M.G.; Lee, K.P.; Choi, H.G.; Kang, S.H.; Klochkova, T.A.; Han, J.W.; Kim, G.H. Characterization of carbohydrate combining sites of Bryohealin, an algal lectin from Bryopsis plumosa. J. Appl. Phycol. 2010, 22, 793-802. [CrossRef]

23. Oliveira, C.; Costa, S.; Teixeira, J.A.; Domingues, L. cDNA cloning and functional expression of the $\alpha$-d-Galactose-Binding lectin frutalin in Escherichia coli. Mol. Biotechnol. 2009, 43, 212-220. [CrossRef] [PubMed]

24. Li, Y.; Liao, X.; Chen, G.; Yap, Y.; Zhang, X. Cloning, expression and purification of Microcystis viridis lectin in Escherichia coli. Mol. Biotechnol. 2011, 47, 105-110. [CrossRef] [PubMed]

25. Adar, R.; Streicher, H.; Rozenblatt, S.; Sharon, N. Synthesis of soybean agglutinin in bacterial and mammalian cells. Eur. J. Biochem. 1997, 249, 684-689. [CrossRef] [PubMed]

26. Stubbs, M.E.; Carver, J.P.; Dunn, R.J. Production of pea lectin in Escherichia coli. J. Biol. Chem. 1986, 261, 6141-6144. [PubMed]

27. Upadhyay, S.K.; Saurabh, S.; Rai, P.; Singh, R.; Chandrashekar, K.; Verma, P.C.; Singh, P.K.; Tuli, R. SUMO fusion facilitates expression and purification of garlic leaf lectin but modifies some of its properties. J. Biotechnol. 2010, 146, 1-8. [CrossRef] [PubMed]

28. Oliveira, C.; Teixeira, J.A.; Domingues, L. Recombinant lectins: An array of tailor-made glycan-interaction biosynthetic tools. Crit. Rev. Biotechnol. 2013, 33, 66-80. [CrossRef] [PubMed]

29. Berlec, A.; Malovrh, T.; Zadravec, P.; Steyer, A.; Ravnikar, M.; Sabotič, J.; Poljšak-Prijatelj, M.; Štrukelj, B. Expression of a hepatitis A virus antigen in Lactococcus lactis and Escherichia coli and evaluation of its immunogenicity. Appl. Microbiol. Biotechnol. 2013, 97, 4333-4342. [CrossRef] [PubMed]

30. Arata, Y.; Hirabayashi, J.; Kasai, K. Sugar binding properties of the two lectin domains of the tandem repeat-type galectin LEC-1 (N32) of Caenorhabditis elegans. Detailed analysis by an improved frontal affinity chromatography method. J. Biol. Chem. 2001, 276, 3068-3077. [CrossRef] [PubMed]

31. Kwon, M.; Jeong, S.; Lee, K.H.; Park, Y.K.; Yu, J. Mimicry of tandem repeat peptides against cell surface carbohydrate. J. Am. Chem. Soc. 2002, 124, 13996-13997. [CrossRef] [PubMed]

32. Skjoedt, M.O.; Roversi, P.; Hummelshoj, T.; Palarasah, Y.; Rosbjerg, A.; Johnson, S.; Lea, S.M.; Garred, P. Crystal structure and functional characterization of the complement regulator mannose-binding lectin (MBL)/ficolin-associated protein-1 (MAP-1). J. Biol. Chem. 2012, 287, 32913-32921. [CrossRef] [PubMed]

33. Sanchez, J.F.; Lescar, J.; Chazalet, V.; Audfray, A.; Gagnon, J.; Alvarez, R.; Breton, C.; Imberty, A.; Mitchell, E.P. Biochemical and structural analysis of Helix pomatia agglutinin. A hexameric lectin with a novel fold. J. Biol. Chem. 2006, 281, 20171-20180. [CrossRef] [PubMed]

34. Fu, P.; Wu, J.; Gao, S.; Guo, G.; Zhang, Y.; Liu, J. The recombinant expression and activity detection of MAF-1 fusion protein. Sci. Rep. 2015, 5. [CrossRef] [PubMed]

35. Senthilkumar, R.; Sharma, K.K. Effect of chaotropic agents on the structure-function of recombinant acylpeptide hydrolase. J. Protein Chem. 2002, 21, 323-332. [CrossRef] [PubMed]

36. Drickamer, K.; Taylor, M.E. Glycan arrays for functional glycomics. Genome Biol. 2002, 3, 1034.1-1034.4. [CrossRef]

37. Oliveira, C.; Teixeira, J.A.; Schmitt, F.; Domingues, L. A comparative study of recombinant and native frutalin binding to human prostate tissues. BMC Biotechnol. 2009, 9, 1-7. [CrossRef] [PubMed]

38. Oliveira, C.; Felix, W.; Moreira, R.A.; Teixeira, J.A.; Domingues, L. Expression of frutalin, an alpha-D-galactose-binding jacalin-related lectin, in the yeast Pichia pastoris. Protein Expr. Purif. 2008, 60, 188-193. [CrossRef] [PubMed]

39. Sato, T.; Tateno, H.; Kaji, H.; Chiba, Y.; Kubota, T.; Hirabayashi, J.; Narimatsu, H. Engineering of recombinant Wisteria floribunda agglutinin specifically binding to GalNAc $\beta$ 1,4GlcNAc (LacdiNAc). Glycobiology 2017, 27, 743-754. [CrossRef] [PubMed]

40. Dunbar, J.; Yennawar, H.P.; Banerjee, S.; Luo, J.; Farber, G.K. The effect of denaturants on protein structure. Protein Sci. 1997, 6, 1727-1733. [CrossRef] [PubMed] 
41. Klochkova, T.A.; Kwak, M.S.; Kim, G.H. Proteomic profiles and ultrastructure of regenerating protoplast of Bryopsis plumosa (Chlorophyta). Algae 2016, 31, 379-390. [CrossRef]

42. Hamid, R.; Masood, A.; Wani, I.H.; Rafiq, S. Lectins: Proteins with diverse applications. J. Appl. Pharm. Sci. 2013, 3, S93-S103.

43. Arenas, M.I.; Romo, E.; De Gaspar, I.; De Bethencourt, F.R.; Sánchez-Chapado, M.; Fraile, B.; Paniagua, R. A lectin histochemistry comparative study in human normal prostate, benign prostatic hyperplasia, and prostatic carcinoma. Glycoconj. J. 1999, 16, 375-382. [CrossRef] [PubMed]

44. Burgess, R.R. Refolding solubilized inclusion body proteins. Methods Enzymol. 2009, 463, 259-279. [PubMed] 